1. Yang CS, Wang X, Lu G, Picinich SC. Cancer prevention by tea: animal studies, molecular mechanisms and human relevance. Nat Rev Cancer. 2009;9(6):429-439.

2. Chow HH, Hakim IA. Pharmacokinetic and chemoprevention studies on tea in humans. Pharmacol Res. 2011;64(2):105-112.

3. Yuan JM, Sun C, Butler LM. Tea and cancer prevention: epidemiological studies. Pharmacol Res. 2011;64(2):123-135.

4. Yang CS, Wang H. Mechanistic issues concerning cancer prevention by tea catechins. Mol Nutr Food Res. 2011;55(6):819-831.

5. Li GX, et al. Pro-oxidative activities and doseresponse relationship of (-)-epigallocatechin-3-gallate in the inhibition of lung cancer cell growth: a comparative study in vivo and in vitro. Carcinogenesis. 2010;31(5):902-910.

6. Leone $\mathrm{M}$, et al. Cancer prevention by tea polyphenols is linked to their direct inhibition of antiapoptotic Bcl-2-family proteins. Cancer Res.
2003;63(23):8118-8121.

7. Urusova DV, et al. Epigallocatechin-gallate suppresses tumorigenesis by directly targeting Pin 1 . Cancer Prev Res. 2011;4:1366-1377.

8. Kumazoe M, et al. 67-kDa laminin receptor increases cGMP to induce cancer-selective apoptosis. JClin Invest. 2013;123(2):787-799.

9. Nelson J, et al. The $67 \mathrm{kDa}$ laminin receptor: structure, function and role in disease. Biosci Rep. 2008;28(1):33-48.

10. Tachibana H, Koga K, Fujimura Y, Yamada K. A receptor for green tea polyphenol EGCG. Nat Struct Mol Biol. 2004;11(4):380-381.

11. Umeda D, Yano S, Yamada K, Tachibana H. Green tea polyphenol epigallocatechin-3-gallate signaling pathway through $67-\mathrm{kDa}$ laminin receptor. J Biol Chem. 2008;283(6):3050-3058.

12. Hong Byun E, et al. TLR4 signaling inhibitory pathway induced by green tea polyphenol epigallocatechin-3-gallate through $67-\mathrm{kDa}$ laminin receptor. JImmunol. 2010;185(1):33-45.
13. Shanafelt TD, et al. Phase 2 trial of daily, oral polyphenon $\mathrm{E}$ in patients with asymptomatic, Rai stage 0 to II chronic lymphocytic leukemia. Cancer. 2013;119(2):363-370.

14. Reagan-Shaw S, Nihal M, Ahmad N. Dose translation from animal to human studies revisited. FASEB J. 2008;22(3):659-661.

15. Klotz T, et al. Vardenafil increases penile rigidity and tumescence in erectile dysfunction patients: a RigiScan and pharmacokinetic study. World J Urol. 2001;19(1):32-39

16. Tsukamoto S, et al. Green tea polyphenol EGCG induces lipid-raft clustering and apoptotic cell death by activating protein kinase Cdelta and acid sphingomyelinase through a $67 \mathrm{kDa}$ laminin receptor in multiple myeloma cells. Biochem J. 2012;443(2):525-534.

17. Scheiman J, Tseng JC, Zheng Y, Meruelo D. Multiple functions of the 37/67-kd laminin receptor make it a suitable target for novel cancer gene therapy. Mol Ther. 2010;18(1):63-74.

\title{
Why stress is BAD for cancer patients
}

\section{Archana S. Nagaraja, ${ }^{1}$ Guillermo N. Armaiz-Pena, ${ }^{1}$ Susan K. Lutgendorf, ${ }^{2}$ and Anil K. Sood ${ }^{1,3,4}$}

1Department of Gynecologic Oncology and Reproductive Medicine, The University of Texas MD Anderson Cancer Center, Houston, Texas, USA

2Department of Psychology, Department of Urology, and Department of Obstetrics and Gynecology, University of lowa, lowa City, Iowa, USA. ${ }^{3}$ Department of Cancer Biology and ${ }^{4}$ Center for RNA Interference and Noncoding RNA,

The University of Texas MD Anderson Cancer Center, Houston, Texas, USA.

\begin{abstract}
Behavioral stress is known to promote tumor progression in experimental models, but the role of behavioral stress in cancer initiation is less clear. In this issue, Hassan et al. focus on the signaling and biological effects induced by stress hormones that lead to tumor cell evasion from apoptosis, resulting in prostate cancer progression.
\end{abstract}

\section{Behavioral stress and cancer}

A stressor is often defined as a stimulus that is capable of activating the hypothalamic-pituitary-adrenal (HPA) axis and/ or the sympathetic nervous system (SNS). HPA activation induces hypothalamic production of neurohormones, such as corticotropin-releasing hormone and vasopressin. This, in turn, results in secretion of the adrenocorticotropic hormone from the pituitary and subsequent release of glucocorticoids from the adrenal cortex. SNS activation results in the release of epinephrine and norepinephrine from sympathetic neurons and adrenal medulla. At a broad level, behavioral stress can be considered as acute or chronic. While acute stress may be adaptive for preservation of the organism and can have beneficial effects (e.g., enhanced immune response), chronic stress can lead to disease states such as car-

Conflict of interest: The authors have declared that no conflict of interest exists.

Citation for this article: J Clin Invest. 2013; 123(2):558-560. doi:10.1172/JCI67887. diovascular and metabolic diseases. Moreover, a growing number of studies have uncovered major roles for chronic stress in cancer progression (1).

Catecholamine-dependent signaling is known to promote several protumoral processes that collectively result in increased tumor progression. For example, chronic stress results in increased epinephrine and norepinephrine, whereas dopamine levels are reduced. This catecholamine shift leads to a microenvironment that is conducive to increased tumor growth and progression in experimental models of disease (2). Specifically, elevated norepinephrine levels have been associated with increased angiogenesis, invasion, and protection from anoikis (2-5). Adrenergic activation has been implicated as the key mediator of these effects by modulating several growth factors (e.g., VEGF, IL-6, IL-8, matrix metalloproteinases, and FAK) in multiple cancers. Upon $\beta$-adrenergic receptor (ADRB) activation, increased cAMP-PKA activity is frequently noted as an intracellular mediator of the stress response.
Chronic stress plays a significant role in cancer progression, and decreased cancer incidence is observed among patients who take beta blockers for the treatment of other diseases (6). Cancer diagnosis and associated treatment can potentially elevate a patient's stress levels, whereas social support has been associated with increased patient survival (7). Recent findings regarding the role of stress hormones in chemoresistance, metastasis, cancer relapse, and surgical recovery have moved the field forward, but the molecular mechanisms underlying these effects are not fully understood $(6,8)$. In this issue of the JCI, Hassan et al. have used a variety of experimental models of prostate cancer in an effort to demonstrate the underlying mechanisms by which behavioral stress promotes tumor growth and to provide the basis to support pharmacological and behavioral interventions for prostate cancer patients (9).

\section{Behavioral stress inhibits tumor cell apoptosis}

The acquired ability of tumor cells to evade apoptosis is a classic hallmark of cancer (10). In advanced prostate cancer, activating antiapoptotic signaling is believed to be an important factor in chemoresistance and androgen-independent tumor growth (11). In the study by Hassan et al., epinephrine 
A

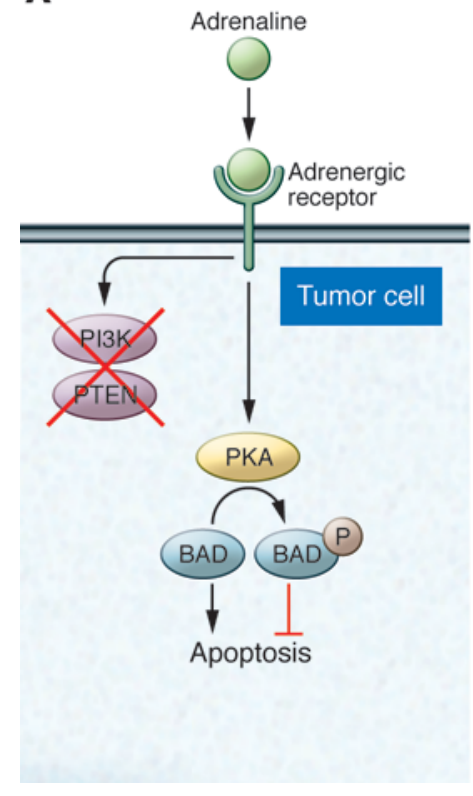

B

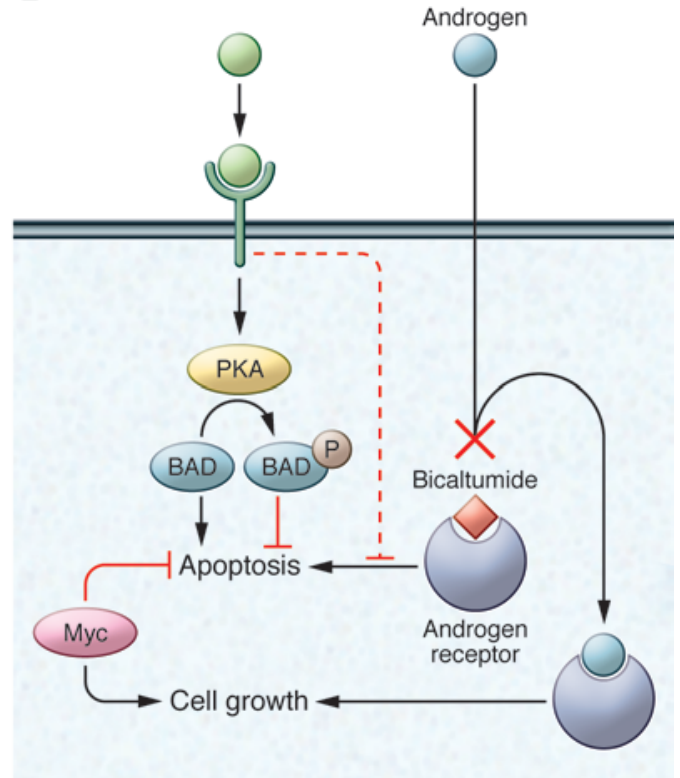

\section{Figure 1}

Hassan et al. explored the role of stress in two models of prostate cancer (9). (A) In an androgen-independent Pten mutant model, signaling through the adrenergic receptor led to phosphorylation (and inactivation) of the proapoptotic molecule BAD, promoting survival of prostate cancer cells. (B) In an androgendependent model, stress signaling blocked apoptosis by the PKA/BAD pathway and contributed to androgen therapy resistance. was found to activate signaling pathways that led to the inhibition of apoptosis (9). The authors used two mouse models of prostate cancer: a prostate-specific, androgendependent Hi-Myc transgenic model, and an androgen-independent PTEN mutant xenograft model (Figure 1). To model behavioral stress, mice were immobilized with or without exposure to predator scent, resulting in markedly elevated epinephrine and norepinephrine levels in the tumor, spleen, and prostate. In both models, stress hormones had an antiapoptotic effect on tumor cells. Additionally, in the PTEN mutant model, epinephrine abrogated the apoptotic effect of a PI3K inhibitor, but this could be restored by delivery of an ADRB2 antagonist. Hassan et al. also provided evidence that PKA could potentially mediate this stress response (9).

The authors found that chronic stress increased the incidence of premalignant lesions in the prostate-specific inducible $\mathrm{Hi}-\mathrm{Myc}$ transgenic mouse model, but this effect was blocked by an ADRB2 inhibitor. Moreover, epinephrine induced activation of ADRB2, resulting in increased PKAmediated BAD phosphorylation and apoptosis inhibition. Importantly, the authors showed that behavioral stress led to resistance to antiandrogen (bicalutamide) therapy, an effect abrogated by treatment with the ADRB2 inhibitor (9). These data support a role for behavioral stress as a promoter of androgen therapy resistance and provide a mechanism that could be potentially targeted to restore sensitivity.
Prostate cancer is associated with various common genetic modifications, including loss of the tumor suppressor genes $p 53$ and PTEN and activation of oncogenes such as c-Jun and c-Myc (12). Additionally, it has been shown that increased Bcl2 levels can lead to the development of androgen-independent prostate cancer (13). This team had previously demonstrated that adrenergic stimulation of a prostate cancer cell line that is PTEN mutant with constitutively active $\mathrm{Bcl} 2$ can protect cells from apoptosis (14). Their present findings further suggest that behavioral stress promotes apoptosis resistance, potentially leading to tumor initiation and progression in androgen-dependent and -independent prostate cancer models (9). Coupled with the authors' previous findings, the current study makes a convincing case for antiapoptotic effects of stress in prostate cancer.

\section{Bench to bedside}

The findings presented in this study have potential clinical implications, suggesting the possibility that beta blockers, generally prescribed to treat high blood pressure or arrhythmia, may increase bicalutamide efficacy. However, although adrenergic activation plays a role in the development of androgen independence, other factors will also confer a survival advantage for cancer cells. These include the activation of the MAPK and AKT signaling pathways, which have been linked to adrenergic signaling in other diseases (13); it will be important to consider these in the development of treatment strategies.

Hassan et al. also demonstrated that immobilization stress accelerated the occurrence of premalignant lesions in the Hi-Myc model (9). Although additional work is needed before there is conclusive proof that behavioral stress can induce cancer onset, these observations provide a fertile ground upon which further studies can be developed to investigate the effect of behavioral stress on cancer initiation.

Clinical studies have shown that in socially isolated ovarian cancer patients, intratumoral levels of norepinephrine were higher than levels in patients with greater social support, whereas the plasma levels were similar between the two groups (15). Here, the authors showed that systemic levels of epinephrine and norepinephrine were higher during stress in the animal models of prostate cancer (9). Furthermore, 12 of 62 prostate cancer patients had elevated epinephrine levels in blood. However, the authors did not show any correlation of blood and intratumor epinephrine levels, outcomes, or progression markers. Ideally, tumor tissue should be used to assess catecholamine levels at the level of the tumor microenvironment, as tissue levels are more likely to adequately reflect catecholamine activity relevant to tumor growth and are likely to be more stable than plasma levels due to the acidic tumor microenvironment. Among the 
important results from this study is the identification of ADRB downstream signaling as a potential predictive factor for patients likely to gain benefit from adjuvant beta blocker therapy while receiving androgen ablation therapy.

\section{Conclusions}

Although the current study provides a new mechanistic understanding of the effects of stress on cancer progression, there remain many unanswered questions with regard to such effects. Among these is the identification of specific patient subsets that are most likely to benefit from interventions targeted against stress-related pathways. Whether such subsets should be identified based on behavioral and/or molecular features is currently unknown. Moreover, identifying reliable downstream markers to test the efficacy of stress-based interventions may allow a more rational selection of therapies. Much like cancer, stress pathways are extremely complex, and it is unclear whether SNS-targeted interventions will be sufficient or whether blocking other pathways, such as HPA mediators or inflammation, will also be required. Addressing these and other questions will be an important component of realizing the full translational potential of the preclinical findings presented in this and other research. Nevertheless, the field continues to rapidly evolve, and novel signaling mechanisms are being discovered that provide a deeper understanding of the effects of behavioral stress on tumor biology. The present study moves the field forward by demonstrating that behavioral stress enables prostate cancer cells to evade apoptosis, an important characteristic in the process of tumor growth and metastasis. In addition, the authors provide a new understanding of mechanisms by which prostate cancer cells could acquire resistance to androgen therapy.

\section{Acknowledgments}

The authors are supported by NIH grants (CA140933, CA104825, CA109298, P50CA083639, P50CA098258, CA128797, U54CA151668), an Ovarian Cancer Research Fund Program Project Development Grant, the Department of Defense (OC073399, W81XWH-10-1-0158, OC100237), the Betty Ann Asche Murray Distinguished Professorship, the Marcus Foundation, and the Blanton-Davis Ovarian Cancer Research Program.

Address correspondence to: Anil K. Sood, Departments of Gynecologic Oncology and Cancer Biology, The University of Texas MD Anderson Cancer Center, Unit 1362, PO Box 301439, Houston, Texas 77230-1439, USA. Phone: 713.745.5266; Fax: 713.792.7586; E-mail: asood@mdanderson.org.

1. Antoni MH, et al. The influence of bio-behavioural factors on tumour biology: pathways and mechanisms. Nat Rev Cancer. 2006;6(3):240-248.

2. Thaker $\mathrm{PH}$, et al. Chronic stress promotes tumor growth and angiogenesis in a mouse model of ovar- ian carcinoma. Nat Med. 2006;12(8):939-944.

3 . Sood AK, et al. Adrenergic modulation of focal adhesion kinase protects human ovarian cancer cells from anoikis. J Clin Invest. 2010;120(5):1515-1523.

4. Sood AK, et al. Stress hormone-mediated invasion of ovarian cancer cells. Clin Cancer Res. 2006;12(2):369-375.

5. Yang EV, et al. Norepinephrine upregulates VEGF, IL-8, and IL-6 expression in human melanoma tumor cell lines: implications for stress-related enhancement of tumor progression. Brain Behav Immun. 2009;23(2):267-275.

6. Armaiz-Pena GN, Cole SW, Lutgendorf SK, Sood AK. Neuroendocrine influences on cancer progression [published online ahead of print June 21, 2012]. Brain Behav Immun. doi:10.1016/j. bbi.2012.06.005.

7. Lutgendorf SK, et al. Social influences on clinical outcomes of patients with ovarian cancer. J Clin Oncol. 2012;30(23):2885-2890.

8. Volden PA, Conzen SD. The influence of glucocorticoid signaling on tumor progression [published online ahead of print November 16, 2012]. Brain Behav Immun. doi:10.1016/j.bbi.2012.10.022.

9. Hassan S, et al. Behavioral stress accelerates prostate cancer development in mice. J Clin Invest. 2013;123(2):874-886

10. Hanahan D, Weinberg RA. The hallmarks of cancer. Cell. 2000;100(1):57-70.

11. McKenzie S, Kyprianou N. Apoptosis evasion: the role of survival pathways in prostate cancer progression and therapeutic resistance. J Cell Biochem. 2006;97(1):18-32

12. Taichman RS, Loberg RD, Mehra R, Pienta KJ. The evolving biology and treatment of prostate cancer. J Clin Invest. 2007;117(9):2351-2361.

13. Feldman BJ, Feldman D. The development of androgen-independent prostate cancer. Nat Rev Cancer. 2001;1(1):34-45.

14. Sastry KS, et al. Epinephrine protects cancer cells from apoptosis via activation of cAMP-dependent protein kinase and BAD phosphorylation. J Biol Chem. 2007;282(19):14094-14100.

15. Lutgendorf SK, et al. Social isolation is associated with elevated tumor norepinephrine in ovarian carcinoma patients. Brain Behav Immun. 2011;25(2):250-255.

\title{
iRHOM2 takes control of rheumatoid arthritis
}

\author{
Stefan F. Lichtenthaler
}

German Center for Neurodegenerative Diseases (DZNE), Munich, Germany. Technische Universität München, Munich, Germany. Munich Cluster for Systems Neurology (SyNergy), Munich, Germany.

\begin{abstract}
The cytokine TNF- $\alpha$ is a major drug target for rheumatoid arthritis, an inflammatory joint disorder. An alternative approach is to target the protease TNF- $\alpha$ convertase (TACE), which releases TNF- $\alpha$ from cells. However, because TACE cleaves other proteins involved in development and cancer, a tissue-specific inhibition of TACE in immune cells appears mandatory. In this issue of the JCI, Issuree et al. report that iRHOM2 is a TACE activator in immune cells. Loss of iRHOM2 largely protects mice from inflammatory arthritis, making iRHOM2 a potential drug target for this condition.
\end{abstract}

Conflict of interest: The author has declared that no conflict of interest exists.

Citation for this article: $J$ Clin Invest. 2013; 123(2):560-562. doi:10.1172/JCI67548.
iPad, iPod, iPhone - iRHOM sounds like the latest gadget you must have. There are even two iRHOM versions. iRHOM1 (also known as RHBDF1) appears to have broad functionality, whereas iRHOM2 (also known as RHBDF2) has more restricted and exclusive functions. iRHOMs are proteolytically inactive homologs of rhomboid proteases. They localize to the membrane of the ER and were initially shown to be part of the ER protein quality control machinery both in Drosophila and mammalian cells (1). Three recent studies demonstrated that an additional function, at least for iRHOM2, is mediating the release of TNF- $\alpha$ from macrophages (2-4). iRHOM2 acts as a cargo receptor 